\title{
Fratura por avulsão da tuberosidade da tíbia em adolescente: Relato de caso*
}

\section{Avulsion Fracture of the Tibial Tuberosity in an Adolescent: Case Report}

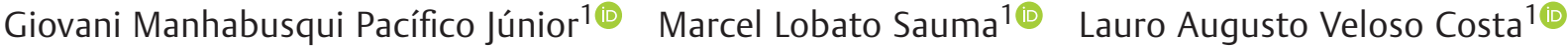 \\ Luiz Fabiano Presente Taniguchi ${ }^{1}$ (i) Francesco Camara Blumetti ${ }^{10}$
}

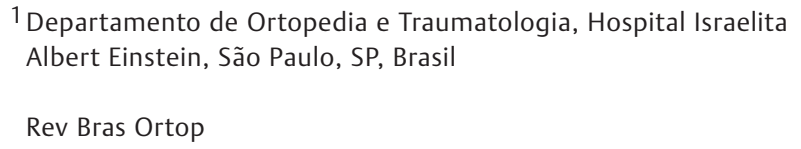

Endereço para correspondência Giovani Manhabusqui Pacífico Júnior, Residente de Ortopedia, Avenida Albert Einstein, 627, Bloco C, Sala 22, Programa Locomotor, Morumbi, São Paulo, SP, 05652-900, Brasil (e-mail: giovani.pacifico@einstein.br).

\section{Resumo \\ Palavras-chave \\ - fraturas da tíbia \\ - fratura por avulsão \\ - traumatismos do joelho \\ O presente estudo tem como objetivo relatar um caso raro de fratura por avulsão da tuberosidade da tíbia em adolescente. Um paciente de 14 anos, do sexo masculino, sofreu entorse de joelho esquerdo durante partida de futebol. No primeiro atendi- mento em pronto-socorro, ele apresentava dor no joelho esquerdo, edema $2+/ 4+$, incapacidade de deambulação e de flexo-extensão do joelho acometido, sem altera- ções neurovasculares ou sinais de síndrome compartimental. Nas radiografias, identi- ficou-se fratura fisária na tíbia proximal esquerda, classificada por Ogden, Tross e Murphy, com modificação por Ryu e Debenham, como tipo IV, e complementada por Aerts et al. como tipo IV-B. Foi realizada imobilização com tala gessada inguino- maleolar e analgesia, e o paciente submetido a cirurgia no dia seguinte, com redução aberta e fixação interna utilizando parafusos canulados $4,5 \mathrm{~mm}$. O paciente recebeu alta no dia seguinte à cirurgia, sendo mantida a imobilização com tala gessada e a restrição de carga por quatro semanas, e apresentou consolidação óssea confirmada por radiografia com três meses do pós-operatório. O paciente evoluiu sem discrepância de comprimento dos membros inferiores, arco de movimento igual ao do membro contralateral, e sem queixas no seguimento de um ano.}

Trabalho realizado no Departamento de Ortopedia e Traumatologia do Hospital Municipal Dr. Moysés Deutsch (M'Boi Mirim), administrado pelo Hospital Israelita Albert Einstein, ambos localizados em São Paulo, SP, Brasil. recebido

19 de Junho de 2020

aceito

16 de Setembro de 2020
DOI https://doi.org/

10.1055/s-0040-1722596. ISSN 0102-3616. (c) 2021. Sociedade Brasileira de Ortopedia e Traumatologia. All rights reserved.

This is an open access article published by Thieme under the terms of the Creative Commons Attribution-NonDerivative-NonCommercial-License, permitting copying and reproduction so long as the original work is given appropriate credit. Contents may not be used for commercial purposes, or adapted, remixed, transformed or built upon. (https://creativecommons.org/ licenses/by-nc-nd/4.0/)

Thieme Revinter Publicações Ltda., Rua do Matoso 170, Rio de Janeiro, RJ, CEP 20270-135, Brazil 


\section{Keywords}

- tibial fractures

- fractures, avulsion

- knee injuries compartment syndrome. Radiographs revealed a physeal fracture at the left proximal tibia, classified by Ogden, Tross and Murphy, and modified by Ryu and Debenham, as type IV, and complemented by Aerts et al. as type IV-B. Immobilization was performed with a plaster cast from the inguinal to malleolar regions, followed by analgesia. The patient was operated on the next day, when open reduction and internal fixation using 4.5-mm cannulated screws were performed. The patient was discharged one day after surgery, with plaster cast immobilization and load restraint for four weeks, and bone consolidation was radiologically confirmed three months after the procedure. The patient evolved with a range of motion similar to that of the contralateral limb, no length discrepancy in the lower limbs, and no complaints after one year of follow-up.

\section{Introdução}

Apesar da frequente exposição do joelho dos adolescentes a traumas violentos, as fraturas por avulsão da epífise proximal da tíbia são infrequentes, variando entre $0,3 \%$ e $2,7 \%$ de todas as lesões fisárias. ${ }^{1-3}$ Os mecanismos de trauma incluem a contração excêntrica do quadríceps no início do salto ou durante aterrisagem, ${ }^{4}$ além de traumas torcionais. ${ }^{5}$ Ocorrem mais frequentemente em adolescentes do sexo masculino com idade entre 14 e 16 anos. ${ }^{1}$ A classificação utilizada para essas fraturas foi descrita por Watson-Jones ${ }^{6}$ em 1955, e modificada por Ogden, Tross e Murphy ${ }^{7}$ e, posteriormente, por Ryu e Debenham. ${ }^{8}$

Este relato apresenta o caso de um adolescente com uma fratura por avulsão atípica da tuberosidade e epífise proximal da tíbia, com seguimento de um ano. A aprovação para o uso dos dados foi obtida do Comitê de Ética e Pesquisa da nossa instituição.

\section{Relato de Caso}

Um paciente de 14 anos, do sexo masculino, sofreu entorse de joelho esquerdo durante partida de futebol. Ele recebeu o primeiro atendimento no pront- socorro de um hospital secundário, e apresentava dor local no joelho esquerdo, edema $2+/ 4+$, incapacidade de deambulação e de flexoextensão, sem alterações neurovasculares e sem sinais de síndrome compartimental. Na série radiográfica, identificou-se fratura fisária na tíbia proximal esquerda (-Figura 1), classificada por Ogden, Tross e Murphy, ${ }^{7}$ com modificação por Ryu e Debenham ${ }^{8}$ como tipo IV, e complementada por Aerts et al. ${ }^{5}$ como tipo IV-B.

Inicialmente, foi realizada a imobilização com tala gessada inguino-maleolar e analgesia. No dia seguinte, o paciente foi submetido a cirurgia com redução aberta e fixação interna utilizando parafusos canulados $4,5 \mathrm{~mm}$ (-Figuras 2A e 2B). 0 paciente recebeu alta no dia seguinte à cirurgia, sendo mantida imobilização com tala gessada e restrição de carga por quatro semanas. Após esse período, ele foi orientado a realizar carga parcial com o auxílio de muletas por duas semanas, e a gradativamente evoluir para carga total junto com fisioterapia para ganho de amplitude de movimento do joelho. O paciente apresentou consolidação óssea confir- mada por radiografia com três meses de pós-operatório (-Figuras 2C e 2D), sendo então liberado para retorno aos esportes. Durante o seguimento de um ano, o paciente evoluiu sem discrepância de comprimento dos membros inferiores e sem desenvolvimento de genu recurvatum, mantendo arco de movimento semelhante ao do membro contralateral (-Figuras 3 e 4 ).

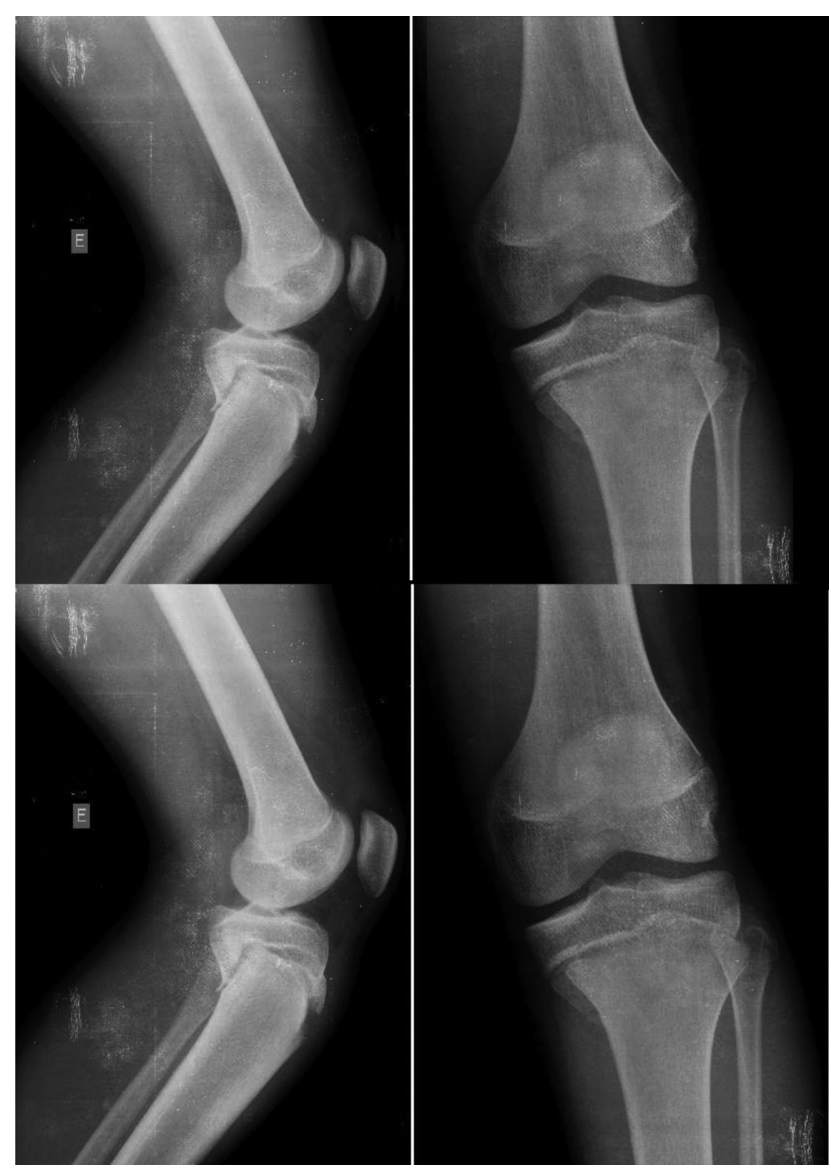

Fig. 1 Radiografias do joelho esquerdo nas incidências anteroposterior (AP) e de perfil evidenciando fratura por avulsão da tuberosidade da tíbia. 


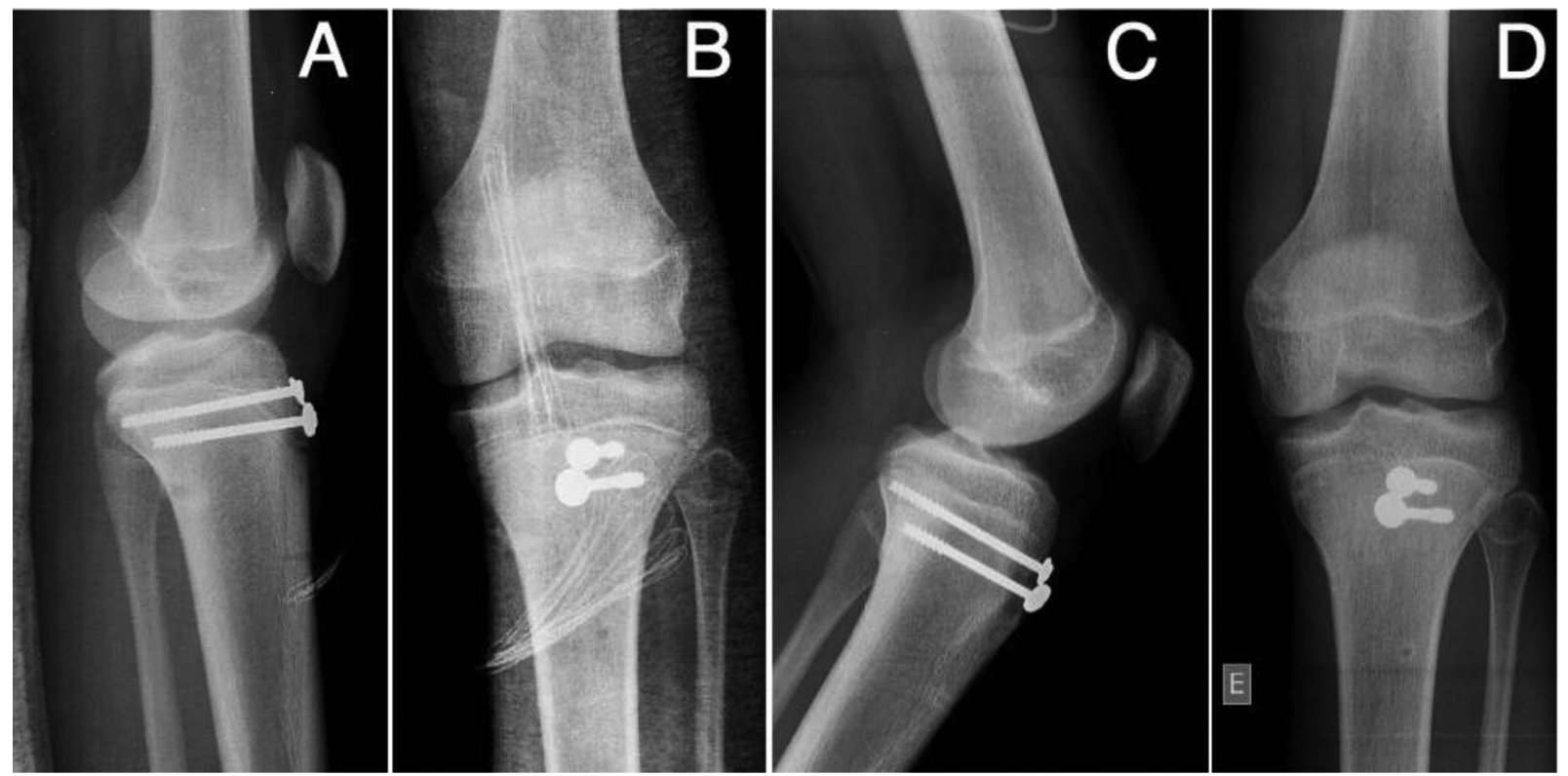

Fig. 2 Radiografias nas incidências AP e de perfil do pós-operatório imediato (A,B); radiografias nas mesmas incidências com três meses de pósoperatório (C,D).

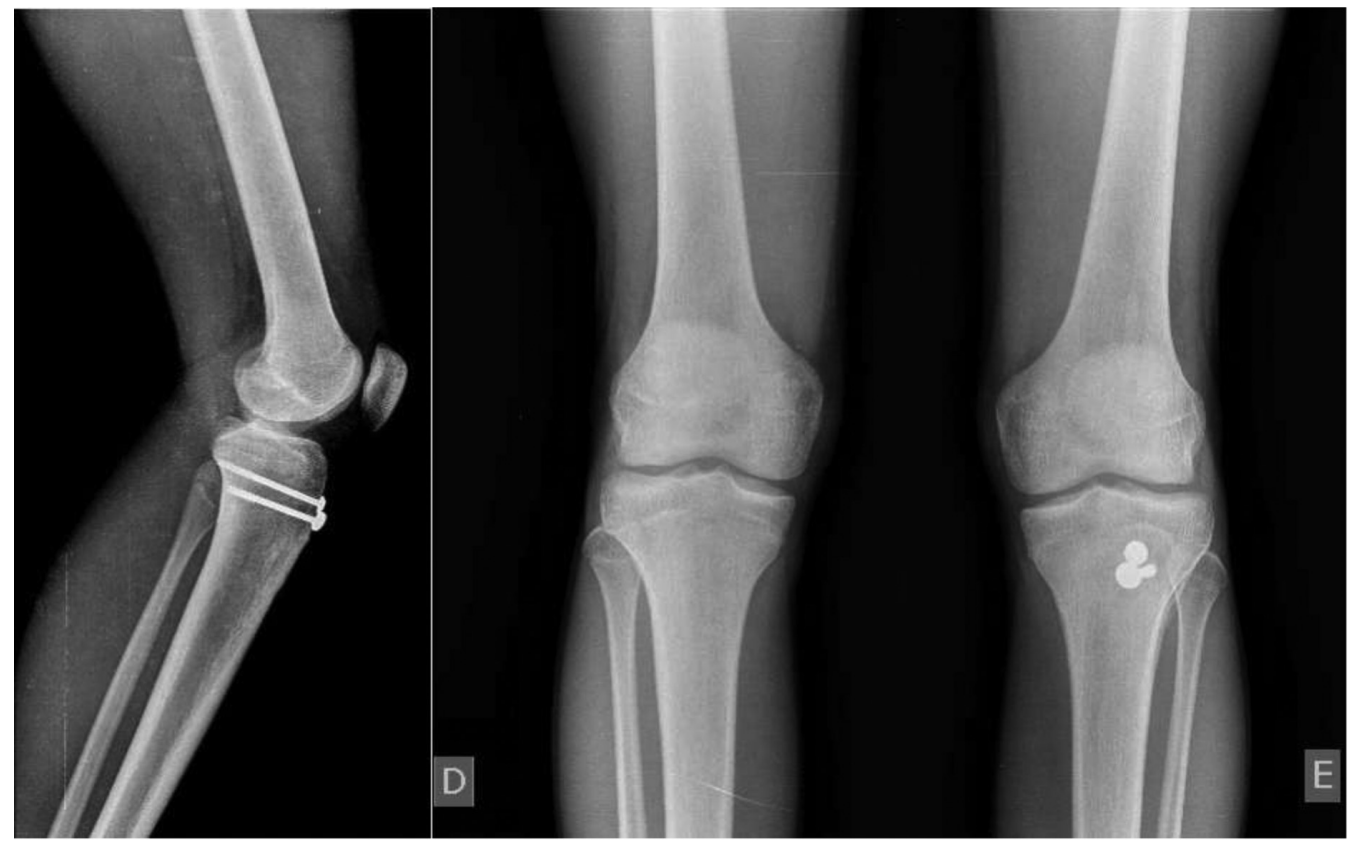

Fig. 3 Radiografias com um ano de pós-operatório.

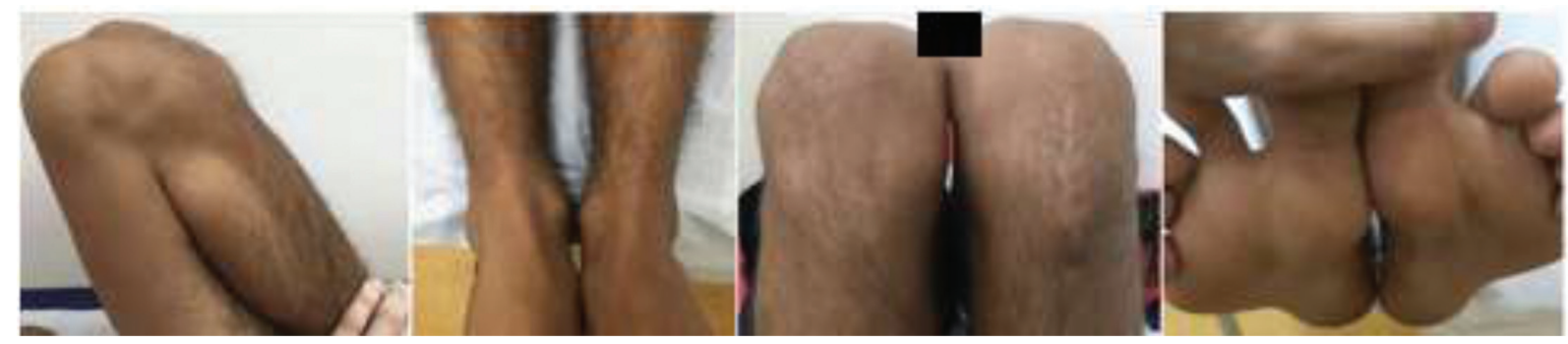

Fig. 4 Imagens clínicas com um ano de pós-operatório. 


\section{Discussão}

Por não apresentar a mesma rigidez do que o tecido ósseo, a fise é uma região do esqueleto imaturo com bastante suscetibilidade a lesões. $\mathrm{O}$ excesso de atividade física aumenta as cargas ao longo da fise, tornando o adolescente atleta mais propenso a lesões da região proximal da tíbia. Alguns dos fatores predisponentes são patela baixa, isquiotibiais encurtados, doença de Osgood-Schlatter preexistente, e anomalias fisárias. $^{9}$

A placa de crescimento é composta por quatro camadas, sendo a zona de células hipertróficas a região mecanicamente mais fraca, na qual geralmente a lesão fisária ocorre. A epífise proximal da tíbia é a segunda maior epífise óssea do corpo, após a epífise distal do fêmur. Sua fragilidade ocorre devido à cartilagem dorsal da apófise (tuberosidade da tíbia), que sofre influência de forças de tração durante toda a fase de ossificação e fusão, ${ }^{5}$ fusão essa que não ocorre de maneira uniforme, pois a região posterior se fecha antes do que a região anterior. ${ }^{2}$ Como a região anterior é mais vulnerável à ação das forças de tração, devido à inserção do tendão patelar, uma fratura por avulsão em um momento em que a região posterior esteja fechada provoca uma fratura metafisária posterior (Salter Harris tipo II), ${ }^{5}$ assim como ocorreu no caso aqui relatado. A raridade dessa lesão pode ser justificada pela pequena inserção ligamentar na região da epífise proximal da tíbia, tornando essa região do osso livre das tensões em varo e valgo às quais o joelho é submetido. ${ }^{3}$

Em 1955, Watson-Jones ${ }^{6}$ classificou as lesões da fise proximal da tíbia em 3 tipos. 0 tipo I é definido como uma avulsão da parte distal da tuberosidade, distal à fise da tíbia proximal. O tipo II prolonga a lesão por toda a fise, mas não atinge a articulação do joelho. $\mathrm{O}$ tipo III é uma avulsão que se estende proximalmente à fise no joelho. ${ }^{6}$ Essa divisão foi modificada por Ogden, Tross e Murphy, ${ }^{7}$ que criaram dois grupos para definir o desvio do fragmento (subtipo A) e a cominuição (subtipo B). Posteriormente, Ryu e Debenham ${ }^{8}$ definiram o tipo IV, descrevendo-o como uma fratura que se estende pela fise e provoca uma avulsão de toda a epífise proximal. Aerts et al. ${ }^{5}$ propuseram a subdivisão do tipo IV em tipo IV-A, quando ocorre apenas a avulsão de toda epífise (Salter Harris I) e tipo IV-B, em que há, além da lesão completa da fise, o acometimento da parte posterior da metáfise, destacando o conhecido fragmento de ThurstonHolland (Salter Harris II), semelhante ao caso apresentado (-Figura 5).

O tratamento dessas lesões apresenta certa dificuldade de redução devido à força constante atuante do quadríceps. Como a maioria dos pacientes estão no período final do crescimento fisário, em geral poucas alterações de crescimento são percebidas. ${ }^{10}$ No caso apresentado, não ocorreram alterações no arco de movimento da articulação e no comprimento do membro. Um protocolo de tratamento aceito é o de Frey et al., ${ }^{4}$ que define o tratamento conservador com imobilização em extensão por quatro a seis semanas nas fraturas do tipo IA, IB e IIA. Os outros tipos são tratados por osteossíntese com redução aberta e fixação interna, seguida por imobilização por quatro a seis semanas, que foi a opção
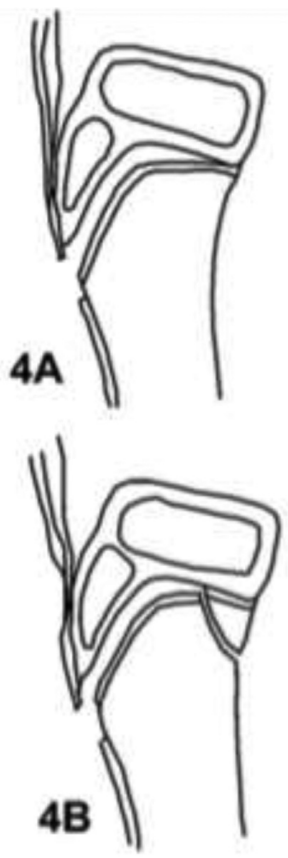

Fig. 5 Classificação tipo IV proposta por Ryu e Debenham, ${ }^{8}$ modificada por Aerts et al. ${ }^{5}$ para IV-B.

escolhida para o caso aqui apresentado. O diagnóstico e tratamento precoce auxiliam nos bons resultados funcionais e no retorno às atividades. ${ }^{10}$

As complicações descritas são síndrome compartimental, lesão dos ligamentos cruzados ou meniscos, ruptura do quadríceps, hipotrofia do quadríceps, hipertrofia ou fratura da tuberosidade da tíbia, calcificação do tendão patelar, discrepância de membros, genu recurvatum, pseudartrose e patela baixa, ${ }^{8}$ sem ocorrência de nenhuma delas no caso aqui relatado.

\section{Conclusão}

A fratura por avulsão da tuberosidade da tíbia, apesar de infrequente e com potencial gravidade devido ao importante acometimento da fise, apresenta bons resultados após tratamento cirúrgico com os métodos usuais. Este artigo apresenta um relato de caso raro de uma fratura do tipo IV-B, de acordo com a modificação de Aerts et al. $^{5}$ Os resultados clínico e funcional foram bons, semelhantes aos descritos na literatura.

\section{Conflito de Interesses}

Os autores declaram não haver conflito de interesses.

\section{Referências}

1 Nanni M, Butt S, Mansour R, Muthukumar T, Cassar-Pullicino VN, Roberts A. Stress-induced Salter-Harris I growth plate injury of the proximal tibia: first report. Skeletal Radiol 2005;34(07): 405-410

2 Mudgal CS, Popovitz LE, Kasser JR. Flexon-type Salter-Harris I injury of the proximal tibial epiphysis. J Orthop Trauma 2000;14 (04):302-305 
3 Rhemrev SJ, Sleeboom C, Ekkelkamp S. Epiphyseal fractures of the proximal tibia. Injury 2000;31(03):131-134

4 Frey S, Hosalkar H, Cameron DB, Heath A, David Horn B, Ganley TJ. Tibial tuberosity fractures in adolescents. J Child Orthop 2008;2 (06):469-474

5 Aerts BR, Ten Brinke B, Jakma TS, Punt BJ. Classification of proximal tibial epiphysis fractures in children: Four clinical cases. Injury 2015;46(08):1680-1683

6 Watson-Jones R. The classic: "Fractures and Joint Injuries" by Sir Reginald Watson-Jones, taken from "Fractures and Joint Injuries," by R. Watson-Jones. Vol. II, 4th ed. Baltimore: Williams and Wilkins Company; 1955. Clin Orthop Relat Res 1974;(105)4-10
7 Ogden JA, Tross RB, Murphy MJ. Fractures of the tibial tuberosity in adolescents. J Bone Joint Surg Am 1980;62(02):205-215

8 Ryu RK, Debenham JO. An unusual avulsion fracture of the proximal tibial epiphysis. Case report and proposed addition to the Watson-Jones classification. Clin Orthop Relat Res 1985; (194):181-184

9 Roy SP, Nag K. Simultaneous bilateral tibial tuberosity avulsion fractures in adolescence: case report and review of 60 years of literature. Injury 2013;44(12):1953-1955

10 Silva Júnior AT, da Silva LJ, da Silva Filho UC, Teixeira EM, Araújo HRS, Moraes FB. Fratura-avulsão tuberosidade anterior da tíbia em adolescente - Relato de dois casos. Rev Bras Ortop 2016;51 (05):610-613 